\title{
Megameatus intact prepuce treated with urethral plate-preserving surgery: a retrospective study of an unusual hypospadias variant
}

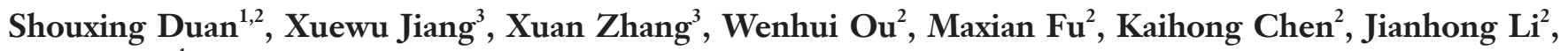 \\ Shuhua $\mathrm{Ma}^{4}$ \\ ${ }^{1}$ Department of Pediatric Surgery, The First Affiliated Hospital of Shantou University Medical College, Shantou 515041, China; ${ }^{2}$ Department \\ of Pediatric Surgery, The Second Affiliated Hospital of Shantou University Medical College, Shantou 515041, China; ${ }^{3}$ Department of Pediatric \\ Surgery, Woman's and Children's Hospital, Shenzhen University and Pingshan District, Shenzhen 518122, China; ${ }^{4}$ Department of Radiology, The \\ First Affiliated Hospital of Shantou University Medical College, Shantou 515041, China \\ Contributions: (I) Conception and design: S Duan, X Jiang, J Li; (II) Administrative support: X Zhang, W Ou, M Fu, K Chen; (III) Provision of study \\ materials or patients: W Ou, M Fu, K Chen; (IV) Collection and assembly of data: X Zhang, W Ou; (V) Data analysis and interpretation: X Zhang, S \\ Duan, S Ma; (VI) Manuscript writing: All authors; (VII) Final approval of manuscript: All authors. \\ Correspondence to: Shuhua Ma. Department of Radiology, The First Affiliated Hospital of Shantou University Medical College, Shantou 515041, \\ China. Email: shuhua6633@163.com; Jianhong Li. Department of Pediatric Surgery, The Second Affiliated Hospital of Shantou University Medical \\ College, Shantou 515041, China. Email: jianhongli2013@163.com.
}

Background: Megameatus intact prepuce (MIP) is a unique variant of hypospadias and is a clinically rare condition. Due to the anatomical characteristics of the MIP hypospadias variant presenting a unique challenge to surgeons, no single urethroplasty method provides a universal solution for all patients. The purpose of this study was to evaluate the outcomes of hypospadias after MIP repair by urethral platepreserving urethroplasty.

Methods: A retrospective study was performed on 25 coronal or distal MIP patients, with a median age of 8 , with most deficiencies being discovered during their first hospital visit for phimosis. Correction with urethroplasty was performed for all patients; 5 underwent the Mathieu procedure, 13 underwent the tubularized incised plate (TIP) procedure, and 7 underwent the Duplay procedure. The 25 patients were followed up for 6 to 36 months to evaluate the surgical outcomes.

Results: There were no significant differences in intraoperative bleeding, hospital stays, postoperative analgesia rate, and cure rate among the three surgical procedures. The operative time for the Mathieu procedure was longer than that for the TIP and Duplay procedures, which did not differ. Complications occurred in 4 of the 25 patients $(16.0 \%)$, and the overall complication-free survival rate at 1 year after surgery was $80.5 \%$. The age at the time of surgery, urethral plate width, urethroplasty length, surgical procedures, or meatal location (coronal or distal penis) were not independently predictive of complications.

Conclusions: The clinical manifestations of MIP are often concealed and then accidentally discovered during hospital visits for phimosis; thus, the actual incidence of MIP might be higher. The urethral plate should be preserved during MIP-correcting treatment, especially for coronal or distal MIP. The same satisfactory outcomes can be obtained with Mathieu, TIP, or Duplay urethroplasty.

Keywords: Duplay; hypospadias; Mathieu; megameatus intact prepuce (MIP); tubularized incised plate urethroplasty (TIP urethroplasty)

Submitted Jun 22, 2019. Accepted for publication Sep 29, 2019.

doi: $10.21037 /$ tau.2019.10.12

View this article at: http://dx.doi.org/10.21037/tau.2019.10.12 


\section{Introduction}

Hypospadias is an abnormality that refers to the urethral opening at the ventral penis, and is due to dysplasia of the ventral tissue of the urethra and penis. The foreskin is abnormally turned towards the dorsal side of the penis and accumulates in a cap-like manner. Hypospadias is one of the major genitourinary congenital malformations observed in male children, with reportedly increasing prevalence (1-3). Megameatus intact prepuce (MIP) is a unique variant of hypospadias and is a clinically rare condition, with an incidence of approximately $1 / 10,000$ that accounts for $1 \%$ to $3 \%$ of hypospadias cases (4). With the increasing prevalence of medical physical examinations, the detection rate of MIP is also rising.

MIP differs from the typical hypospadias. Its anatomical features are as follows: intact foreskin, wide and fish mouthlike urethral opening, deep navicular fossa, wide and shovel-like glans, and no ventral downward curvature or slight dorsal bending of the penis body (5). According to the position of the urethral opening, MIP can be divided into glans, coronal, and distal penis types (5). Due to the anatomical particularity of MIP, it is necessary for clinicians to design a suitable surgical method that takes into account the development of the glans, the width of the urethral plate, the depth of the urethral groove, and the shape and position of the urethral opening to achieve satisfactory therapeutic effects (4-6). No single urethroplasty method provides a universal solution for all patients.

Because the anatomical characteristics of the MIP hypospadias variant present a unique challenge to surgeons for correction (6), increasingly more articles have been focusing on this rare condition in recent years, and awareness and surgical management of MIP continue to evolve $(7,8)$. Although various techniques have been proposed as the "ideal repair" of the MIP hypospadias variant, none has proven superior, and no study has specifically compared the common techniques for treating MIP until now. Therefore, the aim of this study was to analyze the techniques, complications, and outcomes of the MIP hypospadias variant.

\section{Methods}

Institutional review board approval for this study was obtained from the Ethics Committee and Institutional Review Board of the authors' institution (No. 2018-39). A retrospective analysis was performed for patients with MIP from January 2010 to January 2018. Detailed information for each case is shown in Table 1. Patient age ranged from 2 to 13 years, with an average age of 8 years. MIP was diagnosed after hospital admittance; 2 cases of MIP were discovered upon physical examination and were due to abnormal position of the urethra, and 25 cases were discovered with phimosis during the examination preceding posthetomy or exposure of the balanus during preputial orifice stenosis. The urethral opening was located in the coronal area in 15 cases, the distal penis in 10 cases, and the glans in 2 cases. The width of the urethral plate was $0.8-1.2 \mathrm{~cm}$, with an average of $1.06 \pm 1.39 \mathrm{~cm}$.

The operative methods included meatal advancement and glanuloplasty (MAGPI, 1 case), the glans approximation procedure (GAP, 1 case), Mathieu (5 cases), tubularized incised plate urethroplasty (TIP, 13 cases), and Duplay (7 cases). Based on the operative methods, the patients were divided into the Mathieu group, TIP group, and Duplay group; because there was only one MAGPI and one GAP patient, they were excluded from the analysis. There was no significant difference in age among the three groups. All patients were treated with antibiotics to prevent infection after surgery, and urethral catheters were in place for 12-14 days.

The patients were followed up for 6 to 36 months. The operative time, intraoperative bleeding, postoperative analgesia rate, and complications including urethral fistula and meatal stenosis were observed in the three groups, and an analysis was conducted to determine how age at surgery, urethral plate width, urethroplasty length, meatal location, and surgical procedure influence complications.

Statistical analysis was performed using the Statistical Package for the Social Sciences (SPSS), version 20.0 (SPSS Inc., IBM Corp., Armonk, NY, USA). The countable data are presented as number (n) and percentage (\%), and were analyzed by the Chi-square and Fischer's exact tests. Measurements are presented as the mean \pm standard deviation (SD), and were analyzed by analysis of variance (ANOVA). Estimated complicationfree survival curves were obtained using the KaplanMeier method. A $\mathrm{P}<0.05$ was considered to indicate statistical significance.

\section{Results}

Of all patients, only one case underwent MAGPI and one underwent GAP, and thus, these were excluded from the 


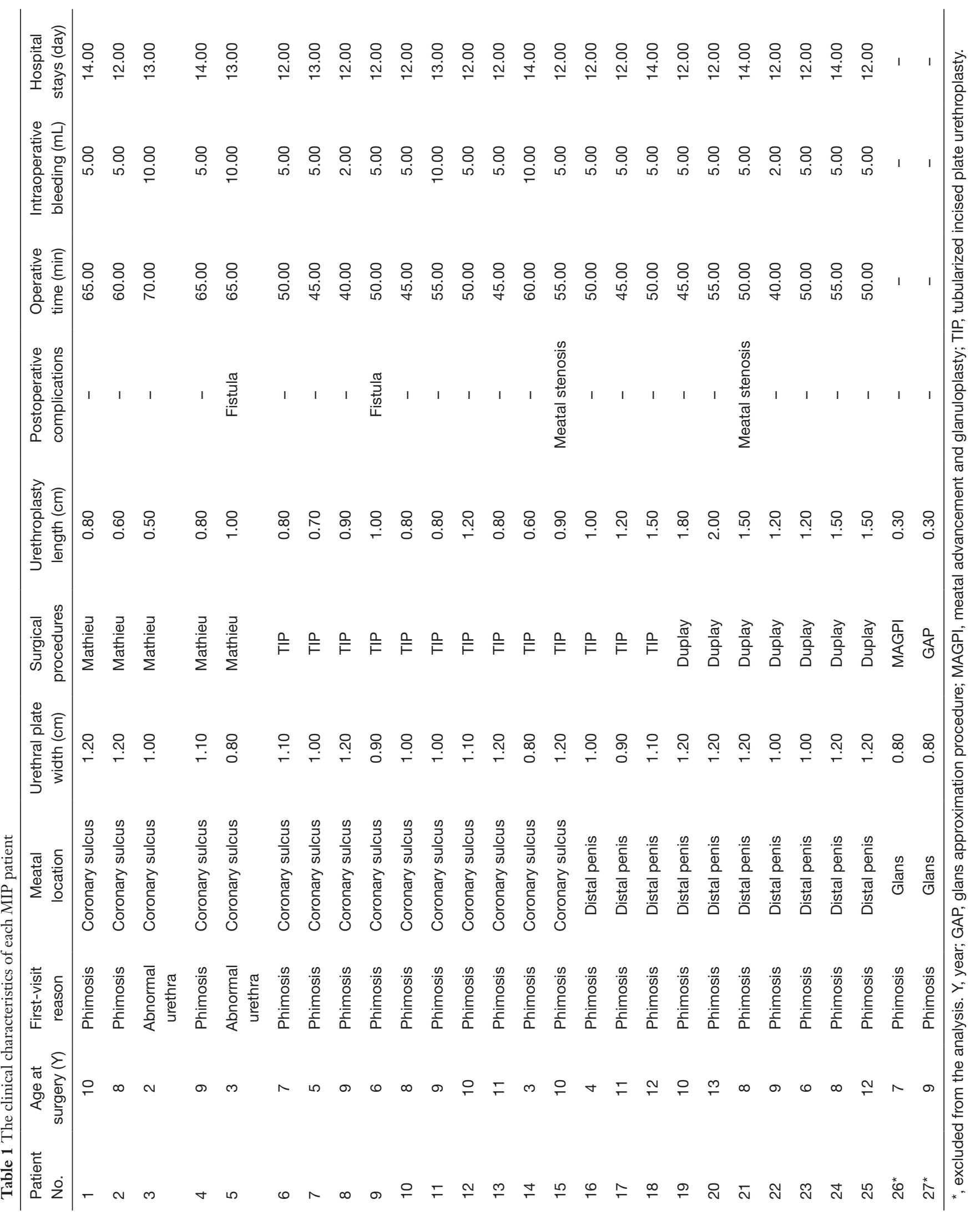


Table 2 Complications after different urethroplasty procedures

\begin{tabular}{lcccccc}
\hline Surgical procedures & Patients & Complications & Fistula & Meatal stenosis & Diverticulum & Infection \\
\hline Mathieu & 5 & 1 & 1 & 0 & 0 & 0 \\
TIP & 13 & 2 & 1 & 1 & 0 & 0 \\
Duplay & 7 & 1 & 0 & 1 & 0 & 0 \\
\hline
\end{tabular}

TIP, tubularized incised plate urethroplasty.

Table 3 Comparison of intraoperative and postoperative management and cure rate in the three groups

\begin{tabular}{lcccccc}
\hline $\begin{array}{l}\text { Surgical } \\
\text { procedures }\end{array}$ & Patients & $\begin{array}{c}\text { Operative } \\
\text { time }(\mathrm{min})\end{array}$ & $\begin{array}{c}\text { Intraoperative } \\
\text { bleeding }(\mathrm{mL})\end{array}$ & $\begin{array}{c}\text { Hospital } \\
\text { stays (day) }\end{array}$ & $\begin{array}{c}\text { Postoperative } \\
\text { analgesia rate }\end{array}$ \\
\hline Mathieu & 5 & $65.00 \pm 3.536^{*}$ & $7.00 \pm 2.738$ & $13.20 \pm 0.836$ & $3 / 5$ & $4 / 5$ \\
TIP & 13 & $49.29 \pm 5.345$ & $5.54 \pm 2.145$ & $12.46 \pm 0.776$ & $7 / 13$ & $11 / 13$ \\
Duplay & 7 & $49.28 \pm 5.345$ & $4.57 \pm 1.134$ & $12.57 \pm 0.976$ & $4 / 7$ & $6 / 7$ \\
P & - & 0.000 & 0.154 & 0.266 & 0.970 & 1.000 \\
\hline
\end{tabular}

Compared with the other two groups, ${ }^{*}, \mathrm{P}<0.05$. TIP, tubularized incised plate urethroplasty.

analysis. Of the remaining 25 cases of coronal or distal penis type, 5 cases underwent the Mathieu urethroplasty procedure, 13 cases underwent the TIP urethroplasty procedure, and 7 cases underwent the Duplay urethroplasty procedure. Complications occurred in 4 of the 25 patients $(16.0 \%)$, including 2 cases of urethral fistula $(8.0 \%)$ and 2 cases of meatal stenosis (8.0\%); 1 case of urethral fistula self-healed, and the remaining 3 cases underwent reoperation. No diverticulum or infection occurred in this group (Table 2).

There were no significant differences in intraoperative bleeding, hospital stay, postoperative analgesia rate, or cure rate among the three groups $(\mathrm{P}>0.05)$. Comparing the Mathieu repair with the TIP or Duplay procedures, statistically significant differences were observed in operation time $(\mathrm{P}<0.05)$. However, there was no significant difference between times for TIP and Duplay procedures $(\mathrm{P}>0.05)$ (Table 3).

Urinary fistula occurred as early as 2 weeks after surgery, and meatal stenosis occurred at 48 weeks after surgery. Figure 1 shows the Kaplan-Meier estimated overall complication-free survival. The primary estimated complication-free survival at 2, 3, 8, and 48 weeks was $96.0 \%, 92.0 \%, 87.8 \%$, and $80.5 \%$, respectively. Primary estimates of Mathieu, TIP, and Duplay complications were $80.0 \%, 84.6 \%$, and $85.7 \%$, respectively. The type of surgical procedure had no significant effect on the prevalence of complications (Figure 2). On univariable analysis, the age at the time of surgery, urethral plate width, urethroplasty length, type of surgical procedure, or meatal location (coronary sulcus or distal penis) did not predict the development of complications (Table 4).

\section{Discussion}

No reports were found documenting MIP accompanied by obvious penile curvature, and most patients are often misdiagnosed at an early age as having phimosis because the foreskin is intact. In Europe or the USA, some cases of MIP are found during circumcision of neonates or in infancy (9). The MIP lesion is hidden and difficult to find. In our study, most cases $(25 / 27,92.6 \%)$ were accidentally discovered during hospital visits for phimosis, with family members being unaware of any urinary tract abnormalities. The actual incidence of MIP might be higher than the current estimates, as a significant number of children with MIP go undetected or are not treated after diagnosis.

There are cases where MIP is not associated with any functional issues. It remains controversial whether patients with partial MIP, but with normal micturition, unobstructed sexual life, and unaffected daily life, must undergo surgical intervention (10). However, surgical correction of MIP in the era of increased cosmetic awareness and concern is 


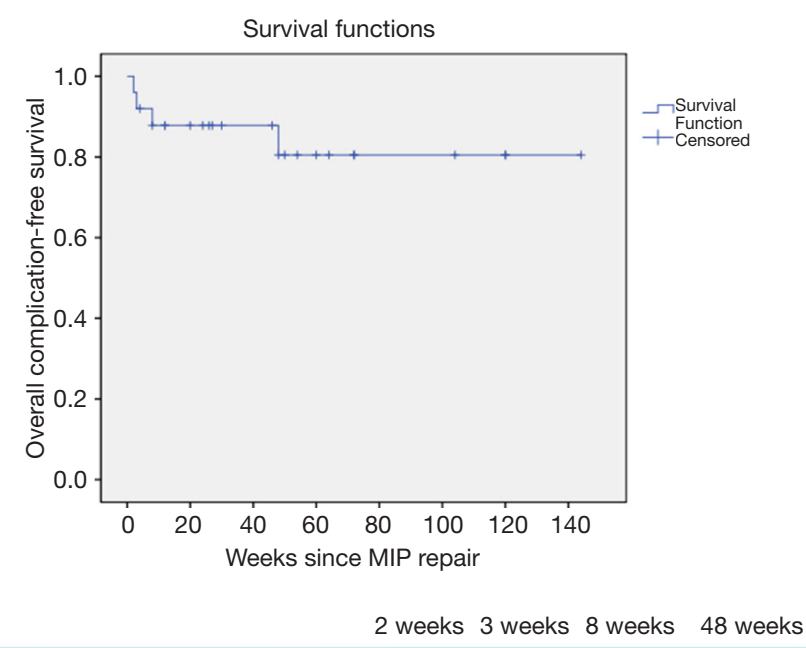

\begin{tabular}{l|llll} 
Overall complication-free survival & $96.0 \%$ & $92.0 \%$ & $87.8 \%$ & $80.5 \%$
\end{tabular}

Figure 1 Kaplan-Meier curve of overall estimated complicationfree survival in 25 patients. MIP, megameatus intact prepuce.

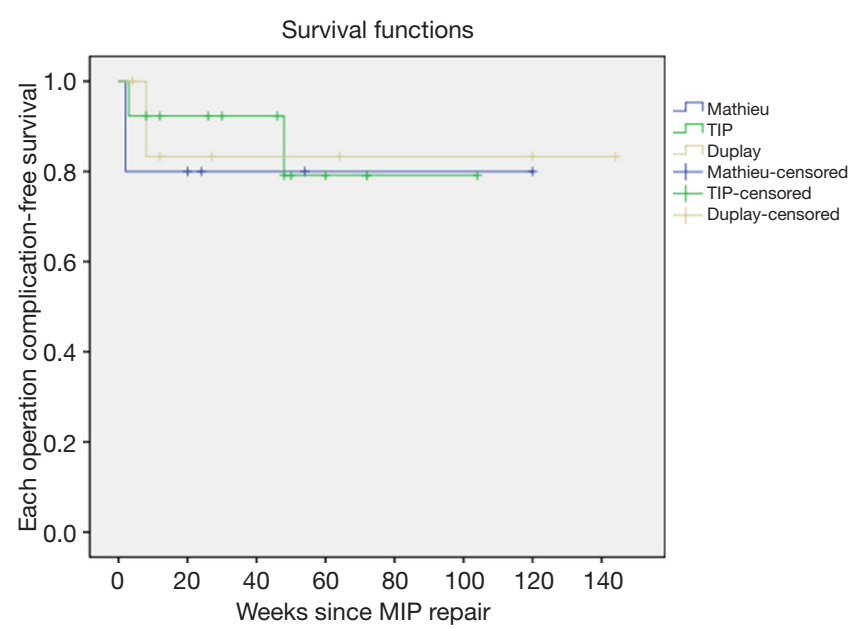

Figure 2 Kaplan-Meier curves of estimated complication-free survival in patients who underwent the Mathieu, TIP, or Duplay surgical procedures. MIP, megameatus intact prepuce; TIP, tubularized incised plate.

Table 4 Analysis of complication predictors

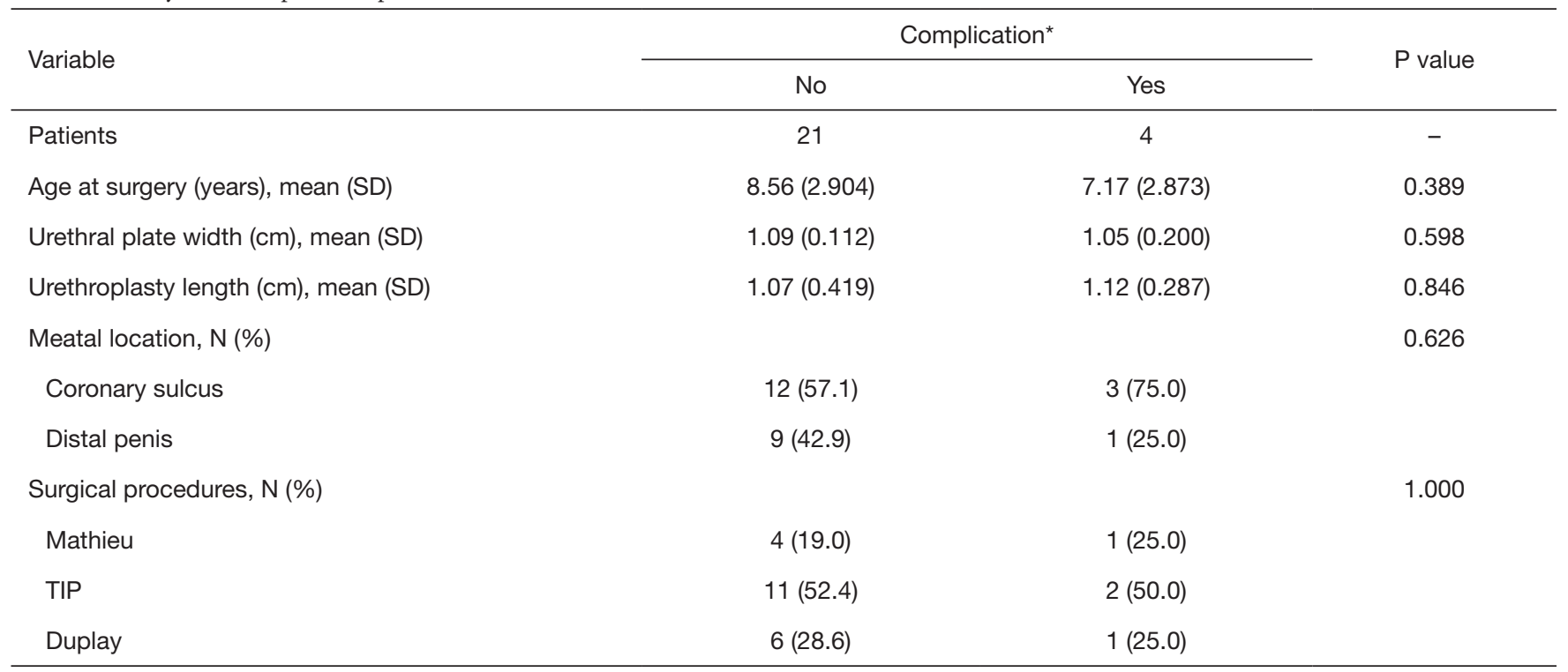

*, excludes two patients with glans. SD, standard deviation; TIP, tubularized incised plate urethroplasty.

justified.

The MIP hypospadias variant is considered a surgical challenge. The suitable procedure for each patient should be tailored according to the anatomic features, and no single technique is appropriate for all variants of MIP hypospadias. Based on the anatomic features, several techniques can be used with good functional and cosmetic results $(5,6,8,10)$, but no data comparing the several common surgical techniques are available. We present our experience with the MIP variants and the common surgical techniques used for treatment, which include the MAGPI, GAP, Mathieu, TIP, and Duplay urethroplasty techniques.

For the glanular type of MIP, the MAGPI or GAP 
procedure can achieve good surgical results in restoring the morphology and function $(5,6)$. These procedures can overcome the challenges of a wide, deep glanular groove and a noncompliant fish mouth. In our series, two patients with glanular MIP underwent MAGPI or GAP surgery. As there was only one case each, these two surgical methods were not included in the following analysis. The remaining 25 cases of coronal or distal MIP underwent Mathieu, TIP, or Duplay surgical procedures.

In the Mathieu technique, the proximal urethral flap is reversed to match the urethral orifice, which retains the distal urethral plate, without distal and proximal urethral anastomosis. This procedure reduces the occurrence of urethral stricture, and the operation is simple and safe (10). Compared to the other two procedures, we found that there was a significantly longer operative time for the Mathieu technique. One of the 5 patients who underwent the Mathieu procedure developed a urinary fistula while removing the urethral catheter at 2 weeks after surgery. The postoperative complication occurred early, which may be related to the reversed proximal urethral flap tissues being too thin, leading to poor blood supply.

The TIP technique is performed by longitudinal incision of the urethral plate to broaden the urethra. The occurrence of urethral stricture or urinary fistula is significantly reduced because the urethral plate is composed of muscle fiber tissue and vascular innervations with no annular anastomosis $(5,6)$. For the 13 patients in the current study who underwent TIP, the fascia flap, with its rich blood supply, was applied to cover the formed urethra, further reducing the incidence of postoperative urinary fistula. Nevertheless, there was still 1 case of urinary fistula after the operation, with the incidence rate being $7.7 \%$ (1/13), which was consistent with the $4-28 \%$ rate reported in previous studies (11-13). The key to the TIP technique is that the midline incision of the urethral plate should be over the corpus cavernosum surface to prevent bleeding, and the urethral plate should be fully expanded to ensure that the urethral suture is tension-free. The use of the subcutaneous fascia to cover the urethra significantly reduces the incidence of urinary fistulae $(13,14)$. In addition, TIP is also suitable for cases in which the initial surgery fails and reoperation is needed (15).

The Duplay technique is a penile skin tube urethroplasty that involves the preservation of a complete urethral plate and the use of the subcutaneous fascia to cover the urethra to prevent postoperative urinary fistula or urethral stricture (5). This procedure is suitable for patients with a wide urethral plate. The urethral plate is acknowledged as the best material for urethral reconstruction (16). Meatal stenosis in one case who underwent the Duplay technique may be associated with urethral scar hyperplasia and contracture.

There are different advantages for each of the three surgical methods. The present results showed that there were no significant differences in intraoperative bleeding, hospital stays, postoperative analgesia rate, and cure rate when comparing the three methods. However, the operative time of the Mathieu procedure was significantly longer than that of the TIP and Duplay procedures, which may be related to the design of the operation and the proficiency of the surgeon. In addition, the complication-free survival analysis indicated that there were no significant differences between the three urethroplasty procedures.

The success of hypospadias surgery is affected by many factors (17). Our current results found that the 1-year complication rate was $19.5 \%$ during the 3 -year follow-up period, which was close to the statistics from a previous report in a large case showing a $24 \%$ complications incidence of distal hypospadias (18). We analyzed the age at surgery, urethral plate width, urethroplasty length, type of surgical procedure, or meatal location (coronal or distal penis), and found that they did not independently predict $c$ omplications.

A common feature of the three surgical procedures is the preservation of the urethral plate. The high success rate of these operations may be due to the normal blood supply to the urethral plate and the urethral covering of fasciae (19), which ensures that the flat urethral plate supported by the sponge can provide a fixed platform for the newly formed urethra. Histological studies have found that the urethral plate, with a rich blood flow supported by the corpus cavernosum, contains abundant vascular smooth muscle, glands, and nerves, and its smooth muscle and connective tissue have strong extensibility. These characteristics are beneficial for urethra reconstruction $(20,21)$.

\section{Conclusions}

The results of the present study showed that the clinical manifestations of MIP are concealed and often accidentally discovered during physician visits for phimosis. Thus, the actual incidence of MIP might be higher than that currently estimated. It is advisable to preserve the urethral plate during correction, especially for coronal or distal MIP. Excluding operative time, the intraoperative and 
postoperative recovery and cure rates were similar between the Mathieu, TIP, and Duplay urethroplasty techniques. In addition, the age at surgery, urethral plate width, urethroplasty length, type of surgical procedure, or meatal location (coronal or distal penis) did not independently predict the development of complications.

\section{Acknowledgments}

Funding: This work was supported by grants from the Postdoctoral Science Foundation of China (grant No. 2019M652990), the Medical Science and Technology Research Foundation of Guangdong Province of China (grant No. A2018060), and the Shantou Technology Bureau Science Foundation of China \{grant No. [2017] 182-34\}.

\section{Footnote}

Conflicts of Interest: The authors have no conflicts of interest to declare.

Ethical Statement: The authors are accountable for all aspects of the work in ensuring that questions related to the accuracy or integrity of any part of the work are appropriately investigated and resolved. Institutional review board approval for this study was obtained from the Ethics Committee and Institutional Review Board of the authors' institution (No. 2018-39).

\section{References}

1. Canon S, Mosley B, Chipollini J, et al. Epidemiological assessment of hypospadias by degree of severity. J Urol 2012;188:2362-6.

2. Hadidi AT. History of hypospadias: Lost in translation. J Pediatr Surg 2017;52:211-7.

3. Keays MA, Starke N, Lee SC, et al. Patient Reported Outcomes in Preoperative and Postoperative Patients with Hypospadias. J Urol 2016;195:1215-20.

4. Duckett JW, Keating MA. Technical challenge of the megameatus intact prepuce hypospadias variant: the pyramid procedure. J Urol 1989;141:1407-9.

5. Mao Y, Tang Y, Chen S, et al. Recognition and management of megameatus with intact prepuce (MIP): a variant of hypospadias. Chin J Pediatr Surg 2011;32:834-6.

6. Bar-Yosef Y, Binyamini J, Mullerad M, et al. Megameatus intact prepuce hypospadias variant: application of tubularized incised plate urethroplasty. Urology 2005;66:861-4.

7. Faasse MA, Dray EV, Cheng EY. Repair of megameatus: a modified approach. J Pediatr Urol 2015;11:100-1.

8. Bhat A, Bhat M, Bhat A, et al. Results of tubularized urethral plate urethroplasty in Megameatus Intact Prepuce. Indian J Urol 2017;33:315-8.

9. Fahmy MAB, Shenawy AAE, Altramsy A, et al. Penile Median Raphe Anomalies as an Indicator of Megameatus Intact Prepuce Anomaly in Children Undergoing Routine Circumcision. Urology 2018;121:164-7.

10. Cendron M. The Megameatus, Intact Prepuce Variant of Hypospadias: Use of the Inframeatal Vascularized Flap for Surgical Correction. Front Pediatr 2018;6:55.

11. Snodgrass WT, Bush N, Cost N. Tubularized incised plate hypospadias repair for distal hypospadias. J Pediatr Urol 2010;6:408-13.

12. Hardwicke JT, Bechar JA, Hodson J, et al. Fistula after single-stage primary hypospadias repair - A systematic review of the literature. J Plast Reconstr Aesthet Surg 2015;68:1647-55.

13. Fahmy O, Khairul-Asri MG, Schwentner C, et al. Algorithm for Optimal Urethral Coverage in Hypospadias and Fistula Repair: A Systematic Review. Eur Urol 2016;70:293-8.

14. Yamataka A, Shimotakahara A, Koga H, et al. Modified Snodgrass tubularized incised plate urethroplasty prevents fistula formation in hypospadias. Pediatr Surg Int 2012;28:919-23.

15. Snodgrass WT, Lorenzo A. Tubularized incised-plate urethroplasty for hypospadias reoperation. BJU Int 2002;89:98-100.

16. Erol A, Baskin LS, Li YW, et al. Anatomical studies of the urethral plate: why preservation of the urethral plate is important in hypospadias repair. BJU Int 2000;85:728-34.

17. van der Horst HJ, de Wall LL. Hypospadias, all there is to know. Eur J Pediatr 2017;176:435-41.

18. Chrzan R, Dik P, Klijn AJ, et al. Quality assessment of hypospadias repair with emphasis on techniques used and experience of pediatric urologic surgeons. Urology 2007;70:148-52.

19. Chatterjee US, Mandal MK, Basu S, et al. Comparative study of dartos fascia and tunica vaginalis pedicle wrap for the tubularized incised plate in primary hypospadias repair. 
BJU Int 2004;94:1102-4.

20. Snodgrass W, Patterson K, Plaire JC, et al. Histology of the urethral plate: implications for hypospadias repair. J Urol 2000;164:988-9.

Cite this article as: Duan S, Jiang X, Zhang X, Ou W, Fu M, Chen K, Li J, Ma S. Megameatus intact prepuce treated with urethral plate-preserving surgery: a retrospective study of an unusual hypospadias variant. Transl Androl Urol 2019;8(6):583590. doi: 10.21037/tau.2019.10.12
21. Bush NC, Snodgrass W. Pre-incision urethral plate width does not impact short-term Tubularized Incised Plate urethroplasty outcomes. J Pediatr Urol 2017;13:625.e1-625.e6. 